\title{
SPECTRAL PERMANENCE FOR JOINT SPECTRA
}

\author{
BY
}

RAUL E. CURTO

\begin{abstract}
For a $C^{*}$-subalgebra $A$ of a $C^{*}$-algebra $B$ and a commuting $n$-tuple $a=\left(a_{1}, \ldots, a_{n}\right)$ of elements of $A$, we prove that $\mathrm{Sp}(a, A)=\mathrm{Sp}(a, B)$, where $\mathrm{Sp}$ denotes Taylor spectrum. As a consequence we prove that $0 \notin \mathrm{Sp}(a, A)$ if and only if
\end{abstract}

$$
\hat{a}=\left(\begin{array}{lll}
d_{1} & & \\
d_{2}^{*} & d_{3} & \\
& d_{4}^{*} & \ddots
\end{array}\right) \in L\left(A \otimes \mathbf{C}^{2^{n-1}}\right)
$$

is invertible, where $d_{i}$ is the $i$ th boundary map in the Koszul complex for $A$. More generally, we show that $\sigma_{\delta, k}(a, A)=\sigma_{\delta, k}(a, B)$ and $\sigma_{\pi, k}(a, A)=\sigma_{\pi, k}(a, B)$ (all $\left.k\right)$, where $\sigma_{\delta}$. and $\sigma_{\pi,}$ are the joint spectra considered by $Z$. Shodkowski.

1. Introduction. Let $A$ be a $C^{*}$-subalgebra of a unital $C^{*}$-algebra $B$ and $1 \in A$. It is well known that for an element $a$ in $A$, the spectrum of $a$ in $A$ coincides with its spectrum in $B$, or, to say it differently, if $A \subset L(H)$ for some Hilbert space $H$, then $a$ is invertible in $A$ iff $a$ is invertible on $H$. This property is called spectral permanence. J. L. Taylor has developed in [8] and [9] a notion of joint invertibility for an $n$-tuple $a=\left(a_{1}, \ldots, a_{n}\right)$ of operators on a Banach space $X$, that relies upon the actions of the $a_{i}$ 's in $X$. The following question arises naturally: assuming that the $a_{i}$ 's are in $A$, is it true that the $n$-tuple $a$ is invertible on $A$ iff $a$ is invertible on $B$ ? (Here, we consider each $a_{i}$ acting on $A$ or $B$ as a left multiplication.) We have-posed this question in [2], where we answered it in the affirmative in case $A$ and $B$ are von Neumann algebras and indicated a proof of the general case when $n=2$.

In this paper we prove the spectral permanence property for general $C^{*}$-algebras and arbitrary $n$. More generally, we consider Słodkowski's joint spectra and show that they also have this property. Our main result is then the following: let $A \subset B$, $a=\left(a_{1}, \ldots, a_{n}\right)$ be an $n$-tuple of elements of $A, E(A, a), E(B, a)$ be the associated Koszul complexes and $\sigma_{\delta, k}(a, A), \sigma_{\delta, k}(a, B), \sigma_{\pi, k}(a, A)$ and $\sigma_{\pi, k}(a, B)$ be the joint spectra considered by Z. Słodkowski [6] (see $\$ 2$ below for a summary of definitions). Then

$$
\begin{aligned}
& \sigma_{\delta, k}(a, A)=\sigma_{\delta, k}(a, B) \\
& \sigma_{\pi, k}(a, A)=\sigma_{\pi, k}(a, B)
\end{aligned} \quad(\text { all } k) .
$$

Received by the editors November 20, 1980 and, in revised form, March 10, 1981.

1980 Mathematics Subject Classification. Primary 47C15, 46L05, 46L10, 47A62.

Key words and phrases. Spectral permanence, joint spectra, von Neumann algebras, universal representation. 
In particular, since the Taylor spectra $\operatorname{Sp}(a, A), \operatorname{Sp}(a, B)$ correspond to the case $k=n$ for both $\sigma_{\delta}$ and $\sigma_{\pi}$ we have

$$
\operatorname{Sp}(a, A)=\operatorname{Sp}(a, B)
$$

in other words, $E(a, A)$ is exact iff $E(a, B)$ is exact.

As a direct consequence of the last identity and the proof of Proposition 3.4 in [2], we show that $a$ is invertible on $A$ if

$$
\hat{a}=\left(\begin{array}{lll}
d_{1} & & \\
d_{2}^{*} & d_{3} & \\
& d_{4}^{*} & \ddots
\end{array}\right) \in L\left(A \otimes \mathbf{C}^{2^{n-1}}\right)
$$

is invertible. Moreover, if $\Phi: A \rightarrow C$ is a *-homomorphism into a $C^{*}$-algebra $C$, then $\sigma_{\delta, k}(\Phi(a), C) \subset \sigma_{\delta, k}(a, A)$ and $\sigma_{\pi, k}(\Phi(a), C) \subset \sigma_{\pi, k}(a, A)$. If $\Phi$ is one-to-one, equality holds.

Given a $C^{*}$-subalgebra $A$ of $L(H)$ ( $H$ a Hilbert space) and a commuting $n$-tuple $a=\left(a_{1}, \ldots, a_{n}\right)$ of elements of $A$, there are two natural Koszul complexes to associate with $a$ : the one on $A$ and the one on $H$. Spectral permanence implies that the exactness of one of them is equivalent to the exactness of the other.

As a final consequence we can show that for $C^{*}$-algebras, the notion of invertibility considered by Taylor is equivalent to the more restrictive one suggested by $R$. Harte [4] (see $\$ 5$ below).

The results in this paper have been announced in [1].

2. Definitions and notations. We shall briefly recall the definition of Taylor spectrum. Let $E^{n}$ be the complex exterior algebra on $n$ generators $e_{1}, \ldots, e_{n}$ with multiplication denoted by $\wedge$. Let $X$ be a Banach space and $a_{1}, \ldots, a_{n}$ be a commuting $n$-tuple of operators on $X$. Define $E_{k}^{n}(X)=E_{k}^{n} \otimes_{\mathbf{C}} X$ and for $k \geqslant 1, D_{k}$ : $E_{k}^{n}(X) \rightarrow E_{k-1}^{n}(X)$ by

$$
D_{k}\left(x \otimes e_{i_{1}} \wedge \cdots \wedge e_{i_{k}}\right)=\sum_{j=1}^{n}(-1)^{j+1} a_{i_{j}} x \otimes e_{i_{1}} \wedge \cdots \wedge \tilde{e}_{i_{j}} \wedge \cdots \wedge e_{i_{k}}
$$

where $\sim$ means deletion. Also define $D_{k}=0$ for $k \leqslant 0$.

It is easily seen that $D_{k} D_{k+1}=0$ for all $k$, that is, $\left\{E_{k}^{n}(X), D_{k}\right\}_{k \in \mathbf{Z}}$ is a chain complex, called the Koszul complex associated with $a$ and $X$ and denoted $E(X, a) . a$ is said to be invertible on $X$ if $E(X, a)$ is exact, i.e., $\operatorname{ker} D_{k}=\operatorname{ran} D_{k+1}$ for all $k$. The Taylor spectrum of $a$ on $X$ is $\operatorname{Sp}(a, X)=\left\{\lambda \in \mathbf{C}^{n}: a-\lambda\right.$ is not invertible $\}$.

Slodkowski has considered in [6] the following more general situation: let $E(X, a)$ be the Koszul complex

$$
0 \rightarrow E_{n}^{n}(X) \stackrel{D_{n}}{\rightarrow} E_{n-1}^{n}(X) \stackrel{D_{n-1}}{\rightarrow} \cdots \stackrel{D_{2}}{\rightarrow} E_{1}^{n}(X) \stackrel{D_{1}}{\rightarrow} E_{0}^{n}(X) \rightarrow 0
$$

and define $E(X, a)$ to be exact at $j$ when $\operatorname{ker} D_{j}=\operatorname{ran} D_{j+1}$. Let $\Sigma_{j}(a, X)=\{\lambda \in$ $\mathrm{C}^{n}: E(X, a-\lambda)$ is not exact at $\left.j\right\}$. Then

$$
\sigma_{\delta, k}(a, X)=\bigcup_{0 \leqslant j \leqslant k} \Sigma_{j}(a, X)
$$


and

$$
\sigma_{\pi, k}(a, X)=\bigcup_{n-k \leqslant j \leqslant n} \Sigma_{j}(a, X) \cup\left\{\lambda \in \mathbf{C}^{n}: \operatorname{ran} D_{n-k}(a-\lambda) \text { is not closed }\right\} .
$$

(The latter is precisely the dual definition of $\sigma_{\delta, k}$.) As special cases one observes that

$$
\operatorname{Sp}(a, X)=\sigma_{\delta, n}(a, X)=\sigma_{\pi, n}(a, X),
$$

and $\sigma_{\delta, 0}(a, X), \sigma_{\pi, 0}(a, X)$ are the right spectrum and approximate point spectrum, respectively. Slodkowski has shown in [6] that the $\sigma_{\delta, k}$ 's and $\sigma_{\pi, k}$ 's have the projection property, the spectral mapping property, the upper semicontinuity property and are nonempty and compact, thus generalizing the basic results of $\mathrm{J}$. L. Taylor, although basing them in Taylor's paper [8] and his joint work with Zelazko [7].

We shall have occasion to use the universal representation associated with a $C^{*}$-algebra $A$. We shall recall here the main facts needed for our work. Let $Q$ be the set of positive continuous linear functionals on $A$. For $f \in Q$ let $\pi_{f}$ be the cyclic representation obtained via the GNS construction. The universal representation $\pi$ : $A \rightarrow L\left(H_{\pi}\right)$ is $\bigoplus_{f \in Q} \pi_{f}$. The weak closure of $\pi(A)$ is denoted $\overline{\pi(A)}$ and called the enveloping algebra for $A$. There is a canonical isometric isomorphism $\varphi$ between $\overline{\pi(A)}$ and $A^{\prime \prime}$ (the second Banach space dual of $A$ ) such that $\varphi \circ \pi=i$, the natural injection of $A$ into $A^{\prime \prime} . \varphi$ is also a homeomorphism between $\overline{(\pi(A)}$, weak operator top.) and ( $A^{\prime \prime}$, weak*-top.). Finally, if $A$ is a $C^{*}$-subalgebra of $B$, and $\pi$ and $\rho$ are the respective universal representations, then $\overline{\pi(A)}$ can be identified with $\overline{\rho(A)}([3, \S 12]$, [5]).

The following form of the polar decomposition for maps between von Neumann algebras will be needed in $\S 3$.

Let $A$ be a von Neumann algebra acting on a Hilbert space $H, k, l \in \mathbf{Z}$ and $A_{k}=A \otimes \mathrm{C}^{k}, A_{l}=A \otimes \mathrm{C}^{l}$. Let $\alpha: A_{k} \rightarrow A_{l}$ be the operator induced by an $l \times k$ matrix over $A$ acting on $A_{k}$ as a left multiplication. If $\alpha=\left(\alpha_{i j}\right)$ let $\alpha^{*}=\left(\alpha_{j i}^{*}\right)$ : $A_{l} \rightarrow A_{k}$. Then $\alpha=v p$, where $v: A_{k} \rightarrow A_{l}$ is a partial isometry (i.e., $v^{*} v$ is a projection) and $p=\left(\alpha^{*} \alpha\right)^{1 / 2} \in M_{k}(A)$. If $\operatorname{im} \alpha, \operatorname{im} v, \operatorname{im} p, \operatorname{ker} \alpha, \operatorname{ker} v$ and $\operatorname{ker} p$ denote the images and kernels of $\alpha, v$ and $p$ (e.g., $\operatorname{im} \alpha=\left\{\alpha a: a \in A_{k}\right\}$ and $\left.\operatorname{ker} \alpha=\left\{a \in A_{k}: \alpha a=0\right\}\right)$, then $\operatorname{im} \alpha \subset \operatorname{im} v$ and $\operatorname{ker} \alpha=\operatorname{ker} v=\operatorname{ker} p$. Also, $\alpha=$ $q w$, where $q=\left(\alpha \alpha^{*}\right)^{1 / 2}, w$ is a partial isometry, $\operatorname{im} \alpha=\operatorname{im} q, \operatorname{ker} \alpha=\operatorname{ker} w$ and $\operatorname{ker} \alpha^{*}=\operatorname{ker} q=\operatorname{ker} w^{*}$.

3. Preliminary facts. The first two lemmas are probably well known. We have not been able to find a reference for the formulation we need here, however, so we shall give their proofs for the sake of completeness.

Lemma 1. Let $A$ be a von Neumann algebra, $k, l \in \mathbf{Z}, A_{k}=A \otimes \mathbf{C}^{k}, A_{l}=A \otimes \mathbf{C}^{\prime}$ and $\alpha: A_{k} \rightarrow A_{l}$ be the operator induced by an $l \times k$-matrix over $A$. Let $\alpha=v p$ be its polar decomposition. Then

(i) im $\alpha$ is closed iff im $p$ is closed.

(ii) $\operatorname{im} p$ closed $\Rightarrow A_{k}=\operatorname{ker} p+\operatorname{im} p$ (algebraic sum).

(iii) $\operatorname{im} \alpha$ closed $\Rightarrow \operatorname{im} \alpha=\operatorname{im} v$. 
Proof. (i) im $p$ closed $\Rightarrow \operatorname{im} \alpha$ closed: assume that $\alpha a_{n}=v p a_{n} \rightarrow b$. Then $p a_{n} \rightarrow$ $v^{*} b$, so that $v^{*} b=p a$ for some $a \in A_{k}$. Also, $v v^{*} \alpha=\alpha$ implies $v v^{*} b=b$. Thus $b=v v^{*} b=v p a=\alpha a$. im $\alpha$ closed $\Rightarrow \operatorname{im} p$ closed: let $\alpha=q w$ be the polar decomposition. Then $\operatorname{im} q(=\operatorname{im} \alpha)$ is closed, so that by the preceding argument im $p$ $\left(=\operatorname{im} \alpha^{*}\right)$ is closed.

(ii) Notice that $p^{1 / 2}$ is always in the closure of $p M_{k}(A)$, so that in the present situation, $p^{1 / 2}=p a$ for some $a$. Then $p=p^{1 / 2} p^{1 / 2}=p^{1 / 2} p a=p p^{1 / 2} a=p p a a=$ $p^{2} a^{2}$. Thus im $p=\operatorname{im} p^{2}$. Given $a_{k} \in A_{k}, p a_{k}=p^{2} b_{k}$ for some $b_{k}$, or $a_{k}-p b_{k} \in$ ker $p$.

(iii) Clearly $\operatorname{im} \alpha \subset \operatorname{im} v$. Let $a \in A_{k}$. By (i) and (ii), $a=b+p c$, with $p b=0$. Then $v a=v b+v p c=\alpha c$, because $\operatorname{ker} v=\operatorname{ker} p$, as desired.

If $X \stackrel{\alpha}{\rightarrow} Y \stackrel{\beta}{\rightarrow} Z$ is a sequence of Banach spaces and bounded operators and $Z^{\prime} \stackrel{\beta^{\prime}}{\rightarrow} Y^{\prime} \stackrel{\alpha^{\prime}}{\rightarrow} X^{\prime}$ is the dual sequence, then $\operatorname{im} \alpha=\operatorname{ker} \beta$ and $\operatorname{im} \beta$ is closed iff $\operatorname{im} \beta^{\prime}=$ $\operatorname{ker} \alpha^{\prime}$ and $\operatorname{im} \alpha^{\prime}$ is closed. Lemma 2 below is a von Neumann algebra analogue.

Lemma 2. Let $A$ be a von Neumann algebra, $k, l, m \in \mathbf{Z}, A_{i}=A \otimes \mathbf{C}^{i}$ for $i=k, l, m$, and $\alpha: A_{k} \rightarrow A_{l}$ and $\beta: A_{l} \rightarrow A_{m}$ be operators induced by matrices over $A$. Let $\alpha^{*}$ and $\beta^{*}$ denote the adjoints in the matrix sense (i.e., $\alpha^{*}=\left(\alpha_{j i}^{*}\right)$ if $\alpha=\left(\alpha_{i j}\right)$ ). The following are equivalent.

(i) $\operatorname{im} \alpha=\operatorname{ker} \beta$ and $\operatorname{im} \beta$ is closed.

(ii) $\operatorname{im} \beta^{*}=\operatorname{ker} \alpha^{*}$ and $\operatorname{im} \alpha^{*}$ is closed.

Proof. Let $\alpha=v p$ and $\beta=q w$ be the polar decompositions. By Lemma 1, we can assume that $\alpha=v$ and $\beta=w$. Suppose that $\operatorname{im} v=\operatorname{ker} w$. Then $w^{*} w-1(\in \operatorname{ker} w)$ is in $\operatorname{im} v$, so that $v v^{*}\left(w^{*} w-1\right)=w^{*} w-1$. Thus $v v^{*}+w^{*} w=1$. It readily follows that $\operatorname{ker} v^{*} \subset \operatorname{im} w^{*}$, the other inclusion being obvious.

Lemma 3. Let $A$ be a $C^{*}$-algebra, $\pi: A \rightarrow L\left(H_{\pi}\right)$ be its universal representation and $a=\left(a_{1}, \ldots, a_{n}\right)$ be a commuting $n$-tuple of elements of $A$ acting on $A$ as left multiplications.

(i) $0 \notin \sigma_{\delta, k}(a, A)$ iff $0 \notin \sigma_{\delta, k}(\pi(a), \overline{\pi(A)})$ (all $\left.k\right)$.

(ii) $0 \notin \sigma_{\pi, k}(a, A)$ iff $0 \notin \sigma_{\pi, k}(\pi(a), \overline{\pi(A)})$ (all $k$ ).

Proof. Let $L_{a_{i}}(i=1, \ldots, n)$ denote the left multiplications induced by the $a_{i}$ 's. Let $\varphi: \overline{\pi(A)} \rightarrow A^{\prime \prime}$ be the canonical isomorphism. Then $0 \notin \sigma_{\delta, k}(a, A)$ iff $0 \notin$ $\sigma_{\delta, k}\left(L_{a}^{\prime \prime}, A^{\prime \prime}\right)$ iff $0 \notin \sigma_{\delta, k}\left(\varphi^{-1}\left(L_{a}^{\prime \prime}\right), \overline{\pi(A)}\right)$. Now $\varphi^{-1}\left(L_{a_{i}}^{\prime \prime}\right) \pi(b)=$ $\varphi^{-1}\left(L_{a_{i}}^{\prime \prime} b\right)=\varphi^{-1}\left(a_{i} b\right)=\pi\left(a_{i} b\right)=\pi\left(a_{i}\right) \pi(b)$, so that $\left.\varphi^{-1}\left(L_{a_{i}}^{\prime \prime}\right)\right|_{\pi(A)}=L_{\pi\left(a_{i}\right)}$. Since both maps are weakly continuous we conclude that $\varphi^{-1}\left(L_{a_{i}}^{\prime \prime}\right)=L_{\pi\left(a_{i}\right)}$. This completes the proof.

4. Spectral permanence. We have shown in [2] that spectral permanence holds for von Neumann algebras and Taylor spectrum. A minor variation of [2, Proposition 3.4 ] gives the result for the $\sigma_{\delta, k}$ 's, while Lemma 2 above is required to deal with the $\sigma_{\pi, k}$ 's. 
Proposition 4. Let $A$ be a von Neumann algebra, $\left\{n_{i}\right\}_{i \in \mathbf{Z}}$ be a sequence of nonnegative integers with $n_{i}=0$ for $i<0, A_{i}=A \otimes \mathbf{C}^{n_{i}}$ and $d_{i}: A_{i} \rightarrow A_{i-1}$ be a linear operator induced by left multiplication by an $\left(n_{i-1} \times n_{i}\right)$-matrix over $A(i \in \mathbf{Z})$. Assume that $d_{i} \circ d_{i+1}=0$ for all $i$ and let $k$ be given. Then the complex

$$
\text { (d): } \cdots \stackrel{d_{k+1}}{\rightarrow} A_{k} \stackrel{d_{k}}{\rightarrow} A_{k-1} \cdots A_{2} \stackrel{d_{2}}{\rightarrow} A_{1} \stackrel{d_{1}}{\rightarrow} A_{0} \rightarrow 0
$$

(i) is exact at $i=0,1, \ldots, k$ iff $l_{i}=d_{i}^{*} d_{i}+d_{i+1} d_{i+1}^{*}$ is invertible (as an operator on $A_{i}$ ) for all $i=0,1, \ldots, k$, and (ii) is exact at $i=n, n-1, \ldots, n-k$ and im $d_{n-k}$ is closed iff $l_{i}=d_{i}^{*} d_{i}+d_{i+1} d_{i+1}^{*}$ is invertible (as an operator on $A_{i}$ ) for all $i=n$, $n-1, \ldots, n-k$.

Proof. (i) follows immediately from [2, Proposition 3.4]. (ii) follows from (i) and Lemma 2.

COROLlary 5. Let $A$ be a von Neumann algebra, $a=\left(a_{1}, \ldots, a_{n}\right)$ be a commuting $n$-tuple of elements of $A, E(A, a)$ be the Koszul complex associated with $A$ and $a$, and $D_{i}$ be the ith boundary map of $E(A, a)$. Then

(i) $0 \notin \sigma_{\delta, k}(a, A)$ iff $D_{i}^{*} D_{i}+D_{i+1} D_{i+1}^{*}$ is invertible (all $\left.i=0,1, \ldots, k\right)$.

(ii) $0 \notin \sigma_{\pi, k}(a, A)$ iff $D_{n-i}^{*} D_{n-i}+D_{n-i+1} D_{n-i+1}^{*}$ is invertible for all $i=0,1, \ldots, k$.

(iii) If $B$ is another von Neumann algebra and $A \subset B$, then

$$
\sigma_{\delta, k}(a, A)=\sigma_{\delta, k}(a, B) \quad \text { and } \sigma_{\pi, k}(a, A)=\sigma_{\pi, k}(a, B) \quad(\text { all } k) \text {. }
$$

Proof. (i) and (ii) are immediate from the proposition. (iii) follows from (i), (ii) and the classical spectral permanence $(n=1)$.

THEOREM 1 (SPECTRAL PERMANENCE). Let $A$ be a $C^{*}$-subalgebra of a unital $C^{*}$-algebra $B, 1 \in A$, and $a=\left(a_{1}, \ldots, a_{n}\right)$ be a commuting $n$-tuple of elements of $A$. Then

(i) $\sigma_{\delta, k}(a, A)=\sigma_{\delta, k}(a, B)($ all $k)$.

(ii) $\sigma_{\pi, k}(a, A)=\sigma_{\pi, k}(a, B)($ all $k)$.

Proof. Let $k$ be given. It suffices to show that $0 \notin \sigma_{\cdot, k}(a, A)$ if and only if $0 \notin \sigma_{\cdot, k}(a, B)$. Let $\pi: A \rightarrow L\left(H_{\pi}\right)$ and $\rho: B \rightarrow L\left(H_{\rho}\right)$ be the universal representations. Then

$$
\begin{array}{rll}
0 \notin \sigma_{\cdot, k}(a, A) \quad \text { iff } & 0 \notin \sigma_{\cdot, k}(\pi(a), \overline{\pi(A)}) \quad \text { (by Lemma 3) } \\
& \text { iff } \quad 0 \notin \sigma_{\cdot, k}(\rho(a), \overline{\rho(A)}) \\
& \text { iff } \quad 0 \notin \sigma_{\cdot, k}(\rho(a), \overline{\rho(B)}) \quad \text { (by Corollary 5(iii)) } \\
\text { iff } & 0 \notin \sigma_{\cdot, k}(a, B) \quad \text { (by Lemma 3). }
\end{array}
$$

5. Some consequences. As in [2], given an $n$-tuple $a=\left(a_{1}, \ldots, a_{n}\right)$ of elements of a $C^{*}$-algebra $A$ we denote by $\hat{a}$ the $2^{n-1}$ by $2^{n-1}$-matrix over $A$

$$
\left(\begin{array}{ccc}
D_{1} & & \\
D_{2}^{*} & D_{3} & \\
& D_{4}^{*} & \cdot
\end{array}\right) .
$$


It is easy to see that if $D_{i} \circ D_{i+1}=0$ for all $i$ (i.e., if $a$ is commutative) then $a$ is invertible iff $L_{i}=D_{i}^{*} D_{i}+D_{i+1} D_{i+1}^{*}$ is invertible for all $i=0,1, \ldots, n$. A combination of this fact and Theorem 1 gives the following corollary.

Corollary 6. Let $A$ be a unital $C^{*}$-algebra, $a=\left(a_{1}, \ldots, a_{n}\right)$ be a commuting $n$-tuple of elements of $A$ and $D_{i}$ be the ith boundary map in the Koszul complex associated with $A$ and $a$. Then $a$ is invertible iff $\hat{a}$ is invertible.

Corollary 7. Let $A$ and $C$ be unital $C^{*}$-algebras, $\Phi: A \rightarrow C$ be a unital *homomorphism and $a=\left(a_{1}, \ldots, a_{n}\right)$ be a commuting $n$-tuple of elements of $A$. Then $\sigma_{\delta, k}(\Phi(a), C) \subset \sigma_{\delta, k}(a, A)$ and $\sigma_{\pi, k}(\Phi(a), C) \subset \sigma_{\pi, k}(a, A)$. If $\Phi$ is one-to-one, there is equality in both containments.

Proof. Let $k$ be given. Define $\Phi_{i}: M_{\left(\begin{array}{c}n \\ i\end{array}\right)}(A) \rightarrow M_{\left(\begin{array}{c}n \\ i\end{array}\right)}(C)$ by $\Phi \otimes$ id. Clearly $\Phi_{i}$ is a *-homomorphism and by Corollary $5,0 \notin \sigma_{\delta, k}(a, A)$ iff $L_{i}(a)=D_{i}^{*}(a) D_{i}(a)$ $+D_{i+1}(a) D_{i+1}^{*}(a)$ is invertible for all $i=0,1, \ldots, k$, so that $L_{i}(\Phi(a))=\Phi_{i}\left(L_{i}(a)\right)$ is invertible for all $i=0,1, \ldots, k$, or $0 \notin \sigma_{\delta, k}(\Phi(a), C)$.

COROllary 8. Let $A$ be a unital $C^{*}$-algebra and $a=\left(a_{1}, \ldots, a_{n}\right)$ be a commuting n-tuple of normal elements of $A$. Then

(i) $0 \notin \mathrm{Sp}(a, A)$ iff $a_{i}^{*} a_{i}+\cdots+a_{n}^{*} a_{n}$ is invertible.

(ii) $E(A, a)$ is exact iff there exists $k, 0 \leqslant k \leqslant n$, such that $\operatorname{ker} d_{k}=\operatorname{im} d_{k+1}$ and im $d_{k}$ is closed.

(iii) $\sigma_{\delta, k}(a, A)=\sigma_{\pi, k}(a, A)=\operatorname{Sp}(a, A)$ for all $k$.

From Theorem 1 above and [2, Proposition 3.4] we immediately get the following fact.

COROllary 9. If $a=\left(a_{1}, \ldots, a_{n}\right)$ is a commuting $n$-tuple of elements of a $C^{*}$ subalgebra $A$ of $L(H)$, then $\sigma_{\delta, k}(a, A)=\sigma_{\delta, k}(a, H)$ and $\sigma_{\pi, k}(a, A)=\sigma_{\pi, k}(a, H)$ for all $k$.

REMARKS. It can easily be shown that under the hypotheses of Proposition 4 for a general $C^{*}$-subalgebra $A$ of $L(H),\left(d_{A}\right)$ is exact iff $\left(d_{L(H)}\right)$ is exact iff $\left(d_{H}\right)$ is exact.

R. Harte has suggested in [4] a definition of joint spectrum more restrictive than Taylor's: a commuting pair $A=\left(A_{1}, A_{2}\right)$ is "invertible in the sense of Joseph Taylor" iff its Koszul complex has an interpolation, or chain homotopy, i.e., iff there exist operators $B_{1}, B_{2}, C_{1}$ and $C_{2}$ such that

$$
\left(C_{1} C_{2}\right)\left(\begin{array}{r}
-A_{2} \\
A_{1}
\end{array}\right)=I ; \quad\left(\begin{array}{r}
-A_{2} \\
A_{1}
\end{array}\right)\left(C_{1} C_{2}\right)+\left(\begin{array}{c}
B_{1} \\
B_{2}
\end{array}\right)\left(A_{1} A_{2}\right)=\left(\begin{array}{cc}
I & 0 \\
0 & I
\end{array}\right) ;
$$

and

$$
\left(A_{1} A_{2}\right)\left(\begin{array}{l}
B_{1} \\
B_{2}
\end{array}\right)=I \text {. }
$$

In [4, Problem 2], he asks whether the new notion is equivalent to Taylor's only for Hilbert spaces. From what we have shown in this note, however, it is enough to take $\left(C_{1} C_{2}\right)=\left(D_{2}^{*} D_{2}\right)^{-1} D_{2}^{*}$ and $\left(\begin{array}{c}B_{1} \\ B_{2}\end{array}\right)=D_{i}^{*}\left(D_{1} D_{1}^{*}\right)^{-1}$ to prove that the two notions agree 
for $C^{*}$-algebras. Harte's definition can of course be generalized to $n$-tuples and again, suitable combinations of the $D_{i}$ 's show that, for $C^{*}$-algebras, it is equivalent to Taylor's nonsingularity. In a sense this justifies our use of the word invertible instead of nonsingular.

We finally mention that an analogue of spectral permanence in the case when $A$ or $B$ are not unital can be easily obtained by using [7, Lemma 1.2].

ACKnowledgement. The author wishes to thank J. Bunce, W. Paschke and N. Salinas for many helpful conversations, and the referee for pointing out an error in the original statement of (ii) of Corollary 8 .

\section{REFERENCES}

1. R. E. Curto, Spectral permanence for joint spectra, Proc. Sympos. Pure Math., 28th Summer Research Inst. in Operator Algebras, Queen's Univ., Kingston, Ontario, 1980 (to appear).

2. , Fredholm and invertible $n$-tuples of operators. The deformation problem, Trans. Amer. Math. Soc. 266 (1981), 129-159.

3. J. Dixmier, Les $C^{*}$-algèbres et leurs representations, Cahiers Scientifiques, vol. 29, Gauthier-Villars, Paris, 1969.

4. R. Harte, Invertibility, singularity and Joseph L. Taylor, preprint.

5. G. K. Pedersen, $C^{*}$-algebras and their automorphism groups, Academic Press, London, 1979.

6. Z. Shodkowski, An infinite family of joint spectra, Studia Math. 61 (1977), 239-255.

7. Z. Slodkowski and W. Zelazko, On joint spectra of commuting families of operators, Studia Math. $\mathbf{5 0}$ (1974), 127-148.

8. J. L. Taylor, A joint spectrum for several commuting operators, J. Funct. Anal. 6 (1970), 172-191.

9. $1-38$. , The analytic functional calculus for several commuting operators, Acta Math. 125 (1970),

Department of Mathematics, University of Kansas, Lawrence, Kansas 66045

Current address: Department of Mathematics, University of Iowa, Iowa City, Iowa 52242 